\title{
A CONCEPT OF ENABLING DATA CONSISTENCY CHECKS BETWEEN PRODUCTION AND PRODUCTION PLANNING USING AI
}

\author{
Emilia Gelwer ${ }^{1}$, Jens Weber ${ }^{2}$ and Frederik S. Bäumer ${ }^{3}$ \\ ${ }^{1}$ Mercedes-Benz AG and University of Paderborn, Fronäckerstr. 40, 71063 Sindelfingen, Germany \\ ${ }^{2}$ Mercedes-Benz AG and University of Applied Sciences Zwickau, Scheffelstr. 39, 08066 Zwickau, Germany \\ ${ }^{3}$ Bielefeld University of Applied Sciences, Interaktion 1, 33619 Bielefeld, Germany
}

\begin{abstract}
A variety of methods and systems must be linked together to support production planning with insights from existing production data. In this short paper, we give insight into our concept intended to show how malfunctions, occurring in running production lines, can be analyzed with AI methods and made available to production planning. Using IoT-enabled production systems, target and live data can be routed via a Manufacturing Service Bus to a data lake and then evaluated on-the-fly using anomaly detection. Detected anomalies are then compared with existing maintenance documentation from an existing ERP system to indicate malfunctions. Stored malfunctions are further evaluated via Natural Language Processing and enriched with information from IoT-enabled production systems. Further analysis of correlating parameters is carried out to store findings in a structured knowledge database to learn for the future from the mistakes of the past.
\end{abstract}

\section{KEYWORDS}

Artificial Intelligence, Anomaly Detection, Production Planning, Production, Data Consistency

\section{INTRODUCTION}

Performing data consistency checks between production and production planning is recommended to increase product quality and reduce costs. In complex industries such as the automotive industry, thousands of resources of any kind are used. Most of the resources have been computerized so that a huge amount of data can be collected. However, the generation of knowledge from raw data and data consistency beyond the borders of different departments remain major challenges. The discrepancies between the actual production area and the planning models due to the continuous optimization process during the production cycles are one of the particular challenges in current information systems (Westkämper/Löffler 2016). There is a research gap when it comes to taking errors from existing production into account in production planning when designing plants, tools, and machines. Inadequate planning lengthens planning activities due to optimization cycles, production errors, and increases maintenance costs. This paper poses the question of whether there is a possibility to process knowledge from current production for future production planning. For this purpose, a concept was developed using established AI and data mining methods that enables data consistency checks between current production data and the planning process of new body-in-white (BIW) plants with a focus on anomaly detection. Sensor data from manufacturing resources as well as maintenance data from production must be taken into account.

\section{RELATED WORK}

Production Planning and Control (PPC) is concerned with ensuring production capacity to meet the business plan to maintain profitability, productivity, and delivery time (Cadavid et al. 2020). PPC operates cross-functionally and can therefore contribute to tremendous improvements in manufacturing. Machine 
learning (ML) approaches are becoming more attractive in manufacturing due to the implementation of Industry 4.0 (I4.0) and the availability of data, high computing performance, and storage capacity (Cadavid et al. 2020).

The concept includes maintenance data as well as real-time production data. Therefore, the "data source management" and "equipment data" that are presented by Tao et al. (2018) have been chosen. The focus lies on "equipment data" due to the main consideration of IoT-devices. Since the aim is to enhance plants during production planning for reduced production downtime, "smart design of products" and "processes" will be selected as a use case (Tao et al. 2018). Due to the transfer of knowledge from production errors to production planning, "knowledge discovery and generation" will be chosen as characteristics of I4.0 (Cadavid et al. 2020). Taking into account "smart design of products and processes" as well as "knowledge discovery and generation" in the area of ML-PPC, the contribution of Kretschmer et al. (2017) and Manns et al. (2015) can be identified. Both consider "management data" but exclude "equipment data". The combination of "knowledge discovery and generation" and the "data source equipment data" reveals literary contributions such as Diaz-Rozo et al. (2017), Li et al. (2018), and Rostami et al. (2018). The identified use cases are related to "smart maintenance or process control" and "monitoring". Related work to the third combination of "equipment data" and "smart design of products and processes" in the area of ML-PPC could not be found (Cadavid et al. 2020).

\section{CONCEPT FOR ENABLING DATA CONSISTENCY CHECKS BETWEEN PRODUCTION AND PRODUCTION PLANNING}

Experts were consulted to identify and evaluate relevant challenges to achieve data consistency checks between production and production planning areas and the identification of use cases from the plants as the first step. The identified use cases can be selected according to various criteria. These criteria include ease of implementation, ease of scaling, competitiveness, or innovative ability. The selected use case is the integration of knowledge concerning incidents from the BIW production process in the early phases of plant design and was analyzed for an automotive manufacturer that has numerous IoT-capable resources in the BIW production line so that a suitable database is available. An additional benefit is expected from the integration of knowledge about malfunctions in the early planning phases, which can save costs.

Most of the integration of AI applications is done through data acquisition and storage, data fusion, and data preparation. It should be determined which data are available and which data are required. Furthermore, the question arises as to where the data comes from, how it can be linked and extracted, which data format is available, and which experts and systems are necessary to achieve data consistency.

The concept is based on the Digital Factory, which enables product development and production planning to be parallelized. The focus is on the earliest stage of the planning process: Rough planning. Rough planning is carried out by the car manufacturer's production planners (Draht 2010). An alphanumeric quantity structure and a 3D layout are created based on a library and which contains single components and part modules. The quantity structure is created manually based on the experience of the production planners. The plant supplier is integrated for the detailed planning (Draht 2010; Walla 2015). On the basis of a specification sheet, the supplier builds up the corresponding resource and optimizes it on the basis of simulation results until the start of production (SOP).

The adaptation of the systems will be continued after the SOP, as some disturbances only occur in the real production environment. In production, IoT-enabled resources send a lot of data in real-time, and a challenge for I4.0 can be the integration of this data (Bauernhansl et al. 2014). This is made possible by a Manufacturing Service Bus (MSB) - an Enterprise Service Bus for production systems (Westkämper et al. 2013). The data transfer is performed via a Message Queue Telemetry Transport (MQTT) protocol in the form of a JavaScript Object Notation (JSON) format.

The concept requires process parameters, but also information from the programmable logic controller (PLC) about errors, messages, and warnings. This information can be processed and extracted via platforms like Elastic Stack. In ERP systems, additional information about malfunctions is provided and this information is documented in natural language by maintenance personnel. 


\subsection{Anomaly Detection and Usage of AI-methods}

An anomaly can be detected based on the process parameters using, for example, Isolation Forest or an AutoEncoder. When an anomaly is detected, it can be categorized and identified as an actual fault by including PLC information such as message type. The integration of the ERP system is also useful because the error descriptions, actions, and causes contained in the messages can be processed using Natural Language Processing (NLP). The identified and described faults can be assigned to individual components from the planning phase. Correlating process parameters from IoT-capable devices are linked with the PLC messages and the ERP documentation, which makes it possible that the fault can be assigned to individual electronic, mechatronic, and mechanical components. By integrating expert knowledge concerning correlating parameters to errors, components that do not send any data can also be considered. In addition to the error descriptions, time data must also be recorded. Thus, the duration of recurring production disturbances can be gathered, which allows the monetary impact of the errors to be indicated. The error information for the respective components in the hierarchical structure is linked to the technologies and product database and stored in a knowledge database. The data is linked by an identification number, which is used in all systems. This is the information concerning resource allocation. The location of the resource is divided into production location, area, building number, production line, PLC area, and station. This information is in turn assigned with its counterparts from the planning library in the concept production planning.

\subsection{Similarity Check and Data Consistency}

When setting up a new plant, the planner chose the resources from the library and assembles his quantity structure. After reaching defined milestones, a similarity check is carried out between the selected resources and the stored resources with faults based on the technology, geometry, load capacity, and other product information. If similar resources were used, a message is sent. The message contains initially only $20 \%$ of most serious faults based on the fault duration. According to the Pareto principle, these account for $80 \%$ of the costs. Furthermore, the planner can communicate the contents of the requirement specification more specifically to the supplier. Besides, the concept of cost planning can be carried out more precisely.

Before the concept can be integrated, individual components testing must be executed. The components are the anomaly detection of the process parameters, the text recognition by NLP of the ERP documentation, the correlation analysis of the different information, the structure of the knowledge database, and the assignment to individual components as well as the provision of knowledge for similarity checks. Initially, the components should be considered individually in parallel. Afterward, the sequential combination of the components to a complete system can be performed to enable data consistency.
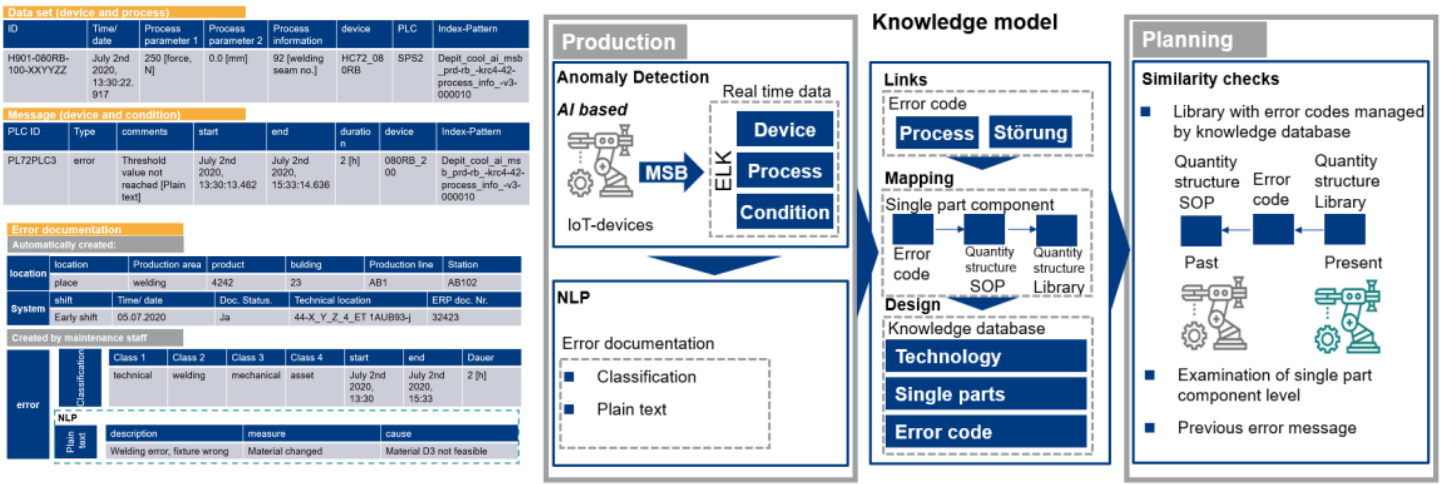

Figure 1. Data set and concept overview 


\section{CONCLUSION}

The short paper presents an early concept for the use of AI methods to improve the detection of anomalies in production planning processes using real production data. Furthermore, the concept can improve knowledge management and data consistency between production and production planning. This way, the production data will be drawn from current assets, processes, and criteria to recognize anomalies and link them with existing error messages. Based on the collected data and the classification of anomalies in the error patterns, AI algorithms can then be developed so that further anomalies can be automatically identified. Various milestones can be compared by performing a comparison of older production lines with various planning statuses based on a similarity check. This will lead to the identification of similar anomalies for the planning process for future production lines. Planning errors will be reduced using a report to the responsible planner.

The concept outlines advantages for the future of structural planning in the automotive industry but also contains limitations. One advantage of considering detected production errors in production planning is to avoid production downtimes and thus to reduce error costs. The knowledge database is continuously updated so that new technologies can be integrated, and the knowledge is transferred independently. Limitations, which could hinder the concept, are currently insufficient documentation of malfunctions by the maintenance staff.

\section{ACKNOWLEDGEMENT}

We thank Rebecca Weber for the discussions. Furthermore, we thank our colleagues from Mercedes-Benz AG for the feedback and ideas for the research project.

\section{REFERENCES}

Bauernhansl T. et al. (pub.) 2014. Industrie 4.0 in Produktion, Automatisierung und Logistik - Anwendung, Technologien, Migration. Springer Vieweg, Wiesbaden.

Cadavid J. et al., 2020. Machine learning applied in production planning and control: a state-of-the-art in the era of industry 4.0. In Journal of Intelligent Manufacturing, Vol. 31, pp. 1531-1558.

Diaz-Roso J. et al., 2017, Machine learning-based CPS for clustering high throughput machining cycle conditions. 45th SME North American manufacturing research conference. Los Angeles, USA, pp. 997-1008.

Draht R. (pub.) 2010. Datenaustausch in der Anlagenplanung mit AutomationML: Integration von CAEX, PLCopen XML and COLLADA. Springer-Verlag, Berlin-Heidelberg.

Kretschmer R. et al., 2017. Knowledge-based design for assembly in agile manufacturing by using data mining methods. In Advanced Engineering Informatics, Vol. 33, pp. 285-299.

Li et al., 2018, Study of SVM-based intelligent dispatcher for parallel machines scheduling with sequence-dependent setup times. $6^{\text {th }}$ international conference on mechanical, automotive and materials engineering, CHAME 2018, Hong Kong, China, pp. 46-50.

Manns M. et al., 2015, Automatic proposal of assembly work plans with a controlled natural language. $9^{\text {th }}$ CIRP conference on intelligent computation in manufacturing engineering. Capri, Italy, pp. 345-350.

Rostami H. et al., 2018. Automatic equipment fault fingerprint extraction for the fault diagnostic on the batch process data. In Applied Soft Computing, Vol. 68, pp. 972-989.

Tao F. et al., 2018. Data-driven smart manufacturing. In Journal of Manufacturing Systems, Vol. 48, pp. 157-169.

Walla W., 2015. Standard- und Modulbasierte digitale Rohbau-Prozesskette: Frühzeitige Produktbeeinflussung bezüglich Produktionsanforderungen im Karosseriebau der Automobilindustrie. Dissertation, Karlsruhe.

Westkämper E., Löffler C. 2016. Strategien der Produktion - Technologien, Konzepte und Wege in die Praxis. Springer Vieweg, Berlin Heidelberg.

Westkämper E. et al. (pub) 2013. Digitale Produktion. Springer Vieweg, Berlin, Heidelberg. 